\title{
Analysis of contributions of herpes simplex virus type 1 UL43 protein to induction of cell-cell fusion
}

\author{
Zhaobing Liu, Junli Huang, Qing Ding, Yan Yang and Hanxiao Sun* \\ Institute of Genomic Medicine, College of Pharmacy, Jinan University, Guangzhou 510632, China \\ *For correspondence: Email: sunhx718@126.com; Tel: +86-020-38375022; Fax: +86-020-38375022 \\ Received: 1 February 2016 \\ Revised accepted: 21 May 2016
}

\begin{abstract}
Purpose: To investigate whether UL43 protein, which is highly conserved in alpha- and gamma herpes viruses, and a non-glycosylated transmembrane protein, is involved in virus entry and virus-induced cell fusion.

Methods: Mutagenesis was accomplished by a markerless two-step Red recombination mutagenesis system implemented on the Herpes simplex virus 1 (HSV-1) bacterial artificial chromosome (BAC). Growth properties of HSV-1 UL43 mutants were analyzed using plaque morphology and one-step growth kinetics. SDS-PAGE and Western blot was employed to assay the synthesis of the viral glycoproteins. Virus-penetration was assayed to determine if UL43 protein is required for efficient virus entry.

Results: Lack of UL43 expression resulted in significantly reduced plaque sizes of syncytial mutant viruses and inhibited cell fusion induced by gBA28 or gKsyn20 $(p<0.05)$. Deletion of UL43 did not affect overall expression levels of viral glycoproteins $g B, g C, g D$, and $g H$ on HSV-1(F) BAC infected cell surfaces. Moreover, mutant viruses lacking UL43 gene exhibited slower kinetics of entry into Vero cells than the parental HSV-1(F) BAC.

Conclusion: Thus, these results suggest an important role for UL43 protein in mediating virus-induced membrane fusion and efficient entry of virion into target cells.
\end{abstract}

Keywords: Herpes simplex virus type 1, UL43 protein, Membrane fusion, Mutant viruses, Virion, Mutagenesis

Tropical Journal of Pharmaceutical Research is indexed by Science Citation Index (SciSearch), Scopus, International Pharmaceutical Abstract, Chemical Abstracts, Embase, Index Copernicus, EBSCO, African Index Medicus, JournalSeek, Journal Citation Reports/Science Edition, Directory of Open Access Journals (DOAJ), African Journal Online, Bioline International, Open-J-Gate and Pharmacy Abstracts

\section{INTRODUCTION}

Herpes simplex virus type 1 (HSV-1) is a common human pathogen that causes most forms of non-genital herpes simplex infection. HSV-1 facilitates virus entry and cell-to-cell spread by mediating fusion which require the intervention of three viral fusogenic glycoproteins, $\mathrm{gB}, \mathrm{gH}$, and $\mathrm{gL}[1,2]$, and occurs downstream of the interaction of $\mathrm{gD}$ with one of the entry receptors [3]. Most mutations that cause extensive virus-induced cell fusion (syncytial or syn mutations) have been mapped to the four HSV genes, UL20 [4], UL24 [5], UL27 encoding glycoprotein B (gB) [6], and UL53 coding for glycoprotein $\mathrm{K}(\mathrm{gK})$ [7]. Little is known of the molecular mechanisms by which HSV regulates its own fusion activity.

UL43 gene of HSV-1 encodes a nonglycosylated transmembrane protein which is conserved only in alpha- and gamma herpes viruses [8]. The HSV-1 UL43 has been shown to be non-essential for virus growth in cell culture and deletion of UL43 did not impair viral replication in a mouse infection model [9]. It has 
been reported that Pseudorabies virus (PrV) UL43 protein strongly inhibited membrane fusion induced by the viral fusion machinery in a transient expression-fusion system [10]. Although the transient co-expression assay does not accurately model viral fusion, it is conceivable that UL43 protein may be involved in modulating membrane fusion.

In this work, we utilized mutant viruses to assess whether the deletion of UL43 gene of HSV-1 affected the ability of dominant syncytial mutations in either $\mathrm{gB}$ or $\mathrm{gK}$ to cause extensive virus-induced cell fusion. We showed that in viral infections UL43 protein is required for virusinduced cell fusion. Moreover, mutant viruses lacking UL43 gene exhibited slower kinetics of entry into Vero cells than the HSV-1(F) BAC, suggesting that UL43 protein functions in membrane fusion phenomena during both virusinduced cell fusion and virion entry.

\section{EXPERIMENTAL}

\section{Cells, virus and plasmids}

All virus mutants derived from $\mathrm{HSV}-1$ strain $\mathrm{F}$ (kindly provided by $\mathrm{Y}$. Mettenleiter, Chicago, IL) were grown on African green monkey (Vero) cells in Dulbecco's modified Eagle's medium (DMEM) supplemented with $10 \%$ fetal calf serum. For transient-complementation experiments, UL43 gene of HSV-1 F was cloned into pCDNA3 plasmid (Life TechnologiesInvitrogen) and named pcDNA-HUL43.

\section{Generation of recombinant viruses}

For generation of HSV-1 $\triangle \mathrm{UL} 43$, the UL43 ORF was mutagenized with synthetic oligonucleotides HUL43-MUT for (5'-TCACGGCCAAGGCGCG GGcTGTGGGGCGTGACGGAACCAAGGATGA CGACGATAAGTAGGG-3') and HUL43-MUT rev (5'-TCCGTCACGCCCCACATAgCCCGCGCCTT GGCCGTGACAACCAATTAACCAATTCTGATT

AG-3'), which mutates the initiation codon from ATG to CTG (shown in lowercase), and HSVAUL43-seq-s (5'-CACAGAATTCGCAAGGC GTTGCTGTCG-3') and HSVAUL43-seq-as (5'GCGGCTCGAGCGCTAAAAAGTTATTT-3').

Using these primers to amplify the AphA1 gene located on pEPkan-S, a PCR product was produced that contained the desired mutation and the kanamycin resistance gene. Mutagenesis was accomplished by using a markerless two-step Red recombination mutagenesis system [11] implemented on the HSV-1 (F) bacterial artificial chromosome (BAC) as described previously $[12,13]$. The desired HSV-1 $\triangle$ UL43 was isolated from non-fluorescent progeny virus plaques. The pHSV1- $\Delta \mathrm{UL} 43$ was used as the backbone for construction of HSV1

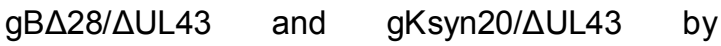
introducing the designated mutations (Figure 1B).

\section{Preparation of antiserum}

The HSV-1 L43 gene product is predicted to possess multiple membrane spanning domains (Figure 1A). We ordered for recombinant membrane protein HSV-1 UL43 (Catalog: MBS1151851) which is His tagged from MyBioSource company. The 43-kDa His-UL43 recombinant protein was purified and used for immunization in a rabbit as described [14].

\section{Virus purification}

Vero cells were infected at 0.001 pfu per cell and incubated for 3 days. The culture medium was harvested and centrifuged at $2000 \mathrm{rpm}$ (Beckman GH3.8) for $20 \mathrm{~min}$ to remove cell debris. Supernatant virions were sedimented by centrifugation for $1 \mathrm{~h}$ at 20,000 rpm (Beckman SW32 rotor) through a $40 \%$ sucrose cushion in phosphate-buffered saline. The clear virus band was harvested and the virions were pelleted at $20,000 \mathrm{rpm}$ for $2 \mathrm{~h}$. The pellets were resuspended in PBS.

\section{Plaque morphology and one-step growth kinetics}

Cell monolayers were infected at $37{ }^{\circ} \mathrm{C}$ for $1 \mathrm{~h}$ and then overlaid with CMC $(0.6 \%$ carboxymethyl cellulose in DMEM + $2 \%$ FCS; Sigma). After incubation at $37{ }^{\circ} \mathrm{C}$ for $48 \mathrm{~h}$, cells were fixed with ice-cold methanol and plaques were visualized after immunohistochemical staining with polyclonal anti-HSV rabbit sera. At $24 \mathrm{~h}$ post transfection, cells were infected with mutant viruses lacking UL43 and incubated as described above. Average plaque sizes and standard deviations were determined and compared to the mean size of plaques induced by HSV-1 (F) BAC, which was set as $100 \%$.

To monitor one-step growth, Vero cells were infected with each virus at a multiplicity of infection (MOI) of 5 and incubated on ice for $1 \mathrm{~h}$. Thereafter, the cells were incubated at $37^{\circ} \mathrm{C}$ and virus was allowed to penetrate for $1 \mathrm{~h}$ at $37^{\circ} \mathrm{C}$. Any remaining extracellular virus was inactivated by low-pH treatment $(\mathrm{pH} 3.0)$, and incubation was continued at $37^{\circ} \mathrm{C}$. At $0,4,12,24,36$, and 
$48 \mathrm{hpi}$, virus stocks were prepared and the virus progeny was titrated on Vero cells.

\section{Quantification of cell-to-cell fusion}

Subconfluent Vero cells in six-well plates were transfected with the plasmid containing T7 RNA polymerase gene (effector cells) or luciferase gene under the control of the T7 promoter (target cells) by using Lipofectamine 2000 (Invitrogen). As positive and negative controls, cells were transfected with both of the plasmids simultaneously and with pCAGGS empty vector plasmid, respectively. Twelve hours post transfection, effector and target cells were detached and seeded in a 24-well plate at a 1:1 ratio.

Twelve hours post seeding, the monolayers were infected with HSV-1 (F) BAC and mutant viruses at an $\mathrm{MOI}$ of 0.2 . At 12 and $24 \mathrm{hpi}$, cells were washed with PBS and lysed with passive lysis buffer. Supernatants were collected and reacted with luciferase substrate (Promega, Madison, $\mathrm{WI})$. The luminescence was measured with a TD20/20 luminometer (Turner Designs).

\section{SDS-PAGE and Western blot assay}

Vero cells were infected with HSV-1 (F) BAC or mutant viruses at a $\mathrm{MOl}$ of 5 . Cells were harvested at $24 \mathrm{hpi}$ and lysed with NP-40 lysis buffer (Life Technologies-Novex). The collected samples were separated by SDS-PAGE (TrisHEPES-SDS gradient 4 to $20 \%$ gels) followed by electrophoretic transfer to nitrocellulose membrane and the presence of various proteins was determined using the primary antibodies described below. Goat anti-mouse secondary antibody conjugated with horseradish peroxidase (HRP) and enhanced chemiluminescence (ECL) substrate were used for detection purposes.

Primary antibodies used were: anti-HSV-1 gB, $\mathrm{gD}, \mathrm{gH}, \mathrm{gL}$ and ICP5 (VP5) monoclonal antibodies (MAbs) (Virusys), anti-HSV-1 gH MAb (Abcam), rabbit anti-HSV-1 UL43 polyclonal antibody (pAb), anti-tubulin alpha (AbD Serotec).

\section{Analysis of membrane-associated proteins}

Biotinylation of cell surface proteins was used to identify membrane-associated proteins of infected Vero cells. Surface proteins of Vero cells infected with the indicated viruses were biotinylated at $12 \mathrm{hpi}$ and isolated with a Pierce cell surface protein isolation kit (Thermo Scientific). The isolated proteins, whole lysates, and flow through were analyzed by Western blots with specific antibodies to $\mathrm{gB}, \mathrm{gD}, \mathrm{gH}, \mathrm{gL}$, UL43, and VP5.

\section{Virus-penetration assay}

Vero cell monolayers were infected at $4{ }^{\circ} \mathrm{C}$ for $1 \mathrm{~h}$ with approximately $250 \mathrm{pfu}$ of HSV-1 (F) BAC and $\triangle U L 43$ mutant viruses. The medium was removed and cells were washed in ice-cold PBS and then incubated at $37{ }^{\circ} \mathrm{C}$ to allow virus penetration. Immediately thereafter (0 $\mathrm{min})$ and at $30,60,120$, and $180 \mathrm{~min}$, unabsorbed virus was inactivated by acid wash. Samples without acid wash served as controls for input virus levels. The cells were overlaid with $\mathrm{CMC}$ and fixed with ice-cold methanol at $48 \mathrm{hpi}$. Plaque numbers were counted for each time point and to determine the entry kinetics, linear regression slopes from 0 to $120 \mathrm{~min}$ were calculated.

\section{Statistical analysis}

All data are expressed as arithmetic mean \pm standard deviation (SD, $\mathrm{n} \geq 3$. Data were processed with GraphPad Prism 5.0, using oneway ANOVA, where appropriate. $P<0.05$ was considered statistically significant.

\section{RESULTS}

\section{Recombinant HSV-1 viruses}

To assess the effect of UL43 on virus-induced cell fusion, we constructed a set of recombinant viruses containing either the gB $\Delta 28$ or gKsyn 20 mutation in the presence or absence of mutations lacking the UL43 gene by using the HSV-1 F genome cloned as a BAC. The set of mutant viruses that were constructed included the following: (i) $\mathrm{gB} \Delta 28$, (ii) $\mathrm{gB} \Delta 28 / \Delta \mathrm{UL} 43$, (iii) gKsyn20, and (iv) gKsyn20/AUL43 (Figure 1B and 2).

\section{Growth properties of HSV-1 UL43 mutants}

Lack of UL43 expression resulted in significantly reduced levels of cell-to-cell fusion and decreased plaque sizes of syncytial mutant viruses (Figure 2A). In contrast, gB- or gKmediated syncytia formation and virus spread were restored to nearly HSV-1 (F) BAC levels (Figure 2B). As expected, deletion of UL43 severely inhibited cell fusion induced by $\mathrm{gB} \Delta 28$ or gKsyn20, exhibiting cell fusion levels lower than those produced by the HSV-1 (F) BAC (Figure 3A, B). One-step growth studies revealed that in Vero cells all viruses replicated in similar manners, achieving viral titers approximately equal to those of the parental virus irrespective of 
A

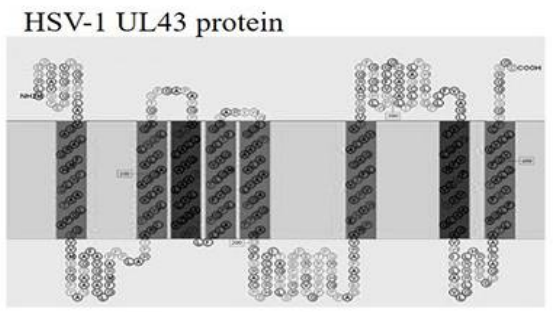

B a

$\stackrel{T R}{\rightarrow}$
PrV UL43 protein

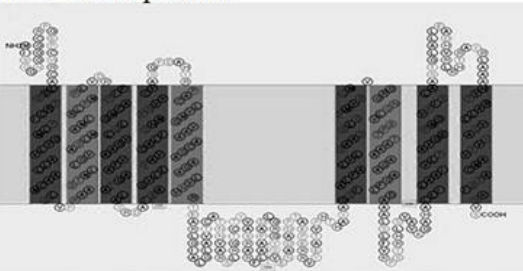

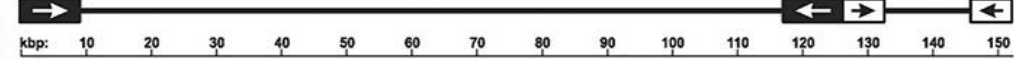

b

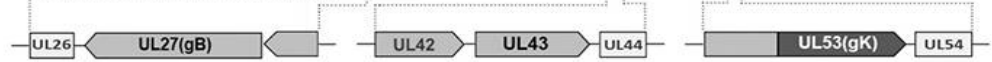

c

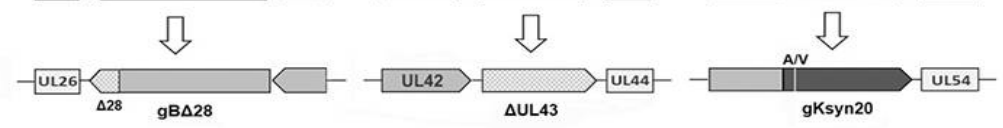

Figure 1: Predicted secondary structure of the HSV-1 UL43 protein and PrV UL43 protein (A). Schematic of the construction of mutant viruses (B)

A

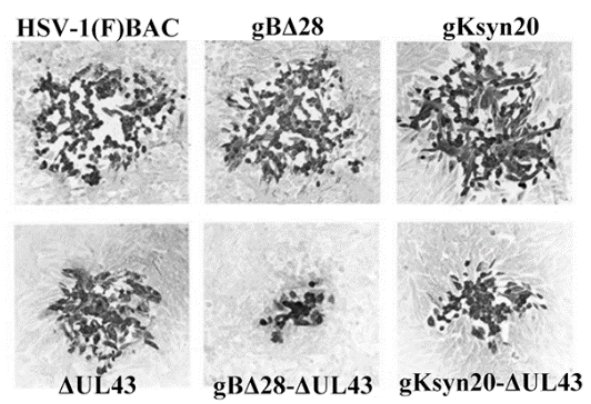

B

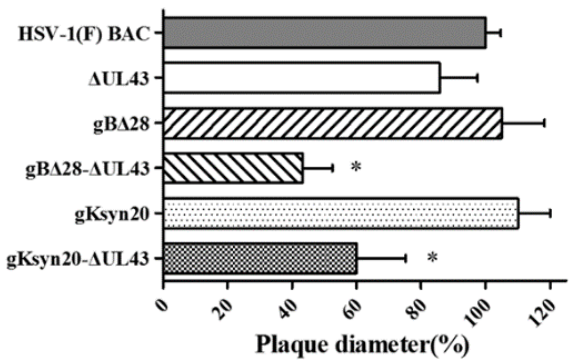

Figure 2: Plaque morphologies and relative sizes of mutant viruses and HSV-1(F) BAC; ${ }^{*} p<0.05$ compared with HSV-1(F) BAC

A

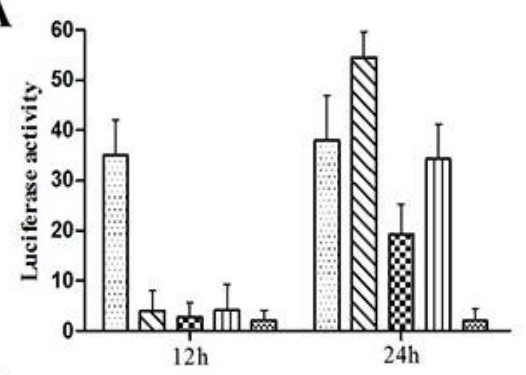

C

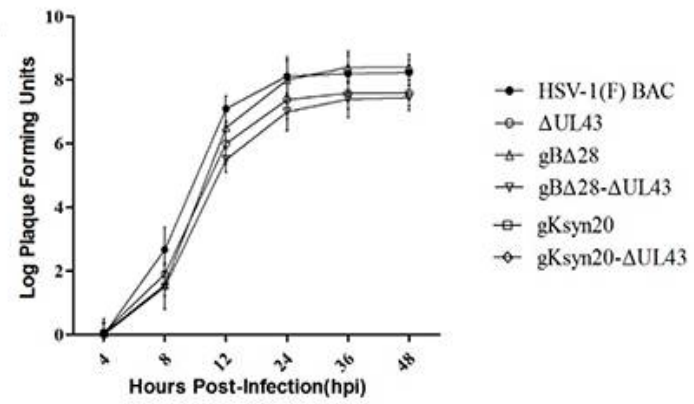

$\square$ Positive control $\triangle \mathrm{gB} \Delta 28$ gBA28-AUL43 曲 gKsyn20

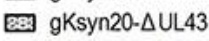
皿 HSV-1(F) BAC Mock
B

D
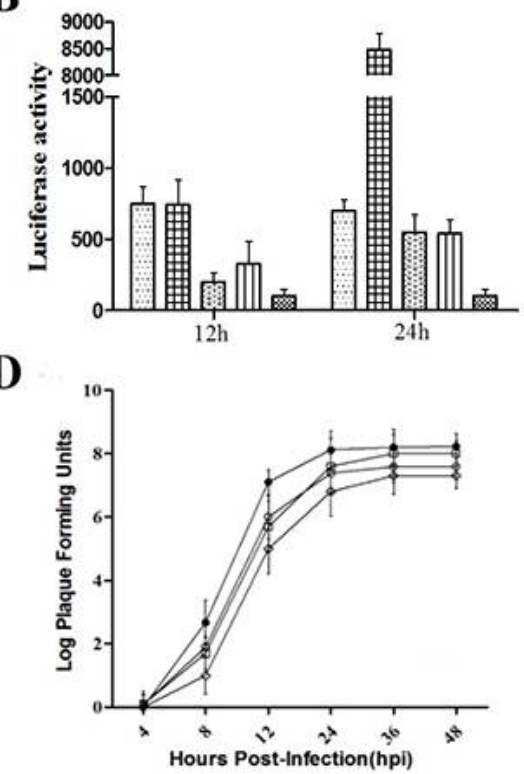

Figure 3: Quantification of virus-induced cell fusion (A-B) and one-step replication kinetics (C-D) 

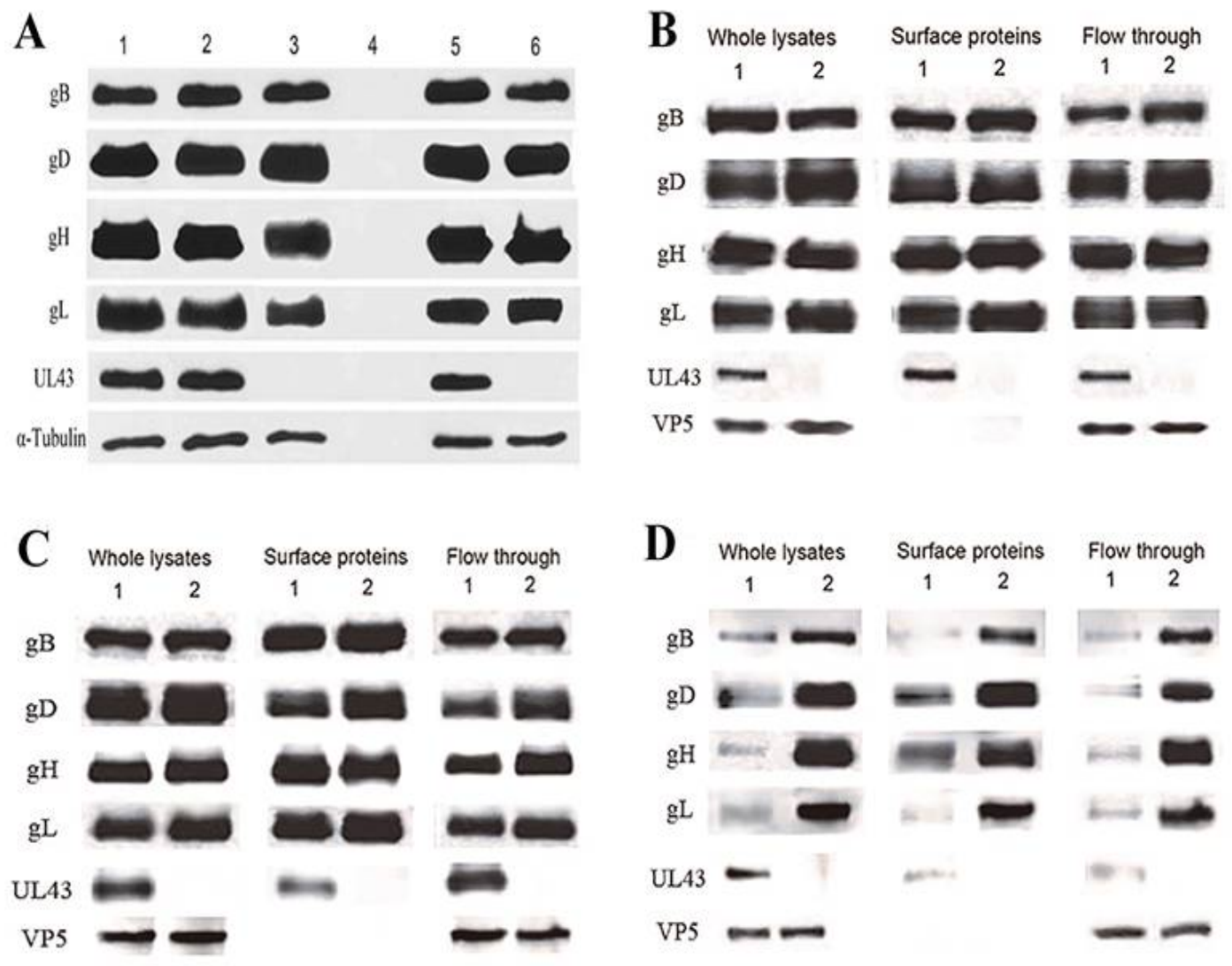

Figure 4: Western blot analysis of viral glycoprotein expression levels in infected Vero cells. A: 1, HSV-1(F) BAC; 2, gB $\Delta 28 ; 3$, gB $\Delta 28 / \Delta U L 43 ; 4$, mock infection; 5, gKsyn20; 6 , gKsyn20/ $\Delta$ UL43. The tubulin signals were used as loading controls. B-D: Whole lysates and membrane-associated proteins were determined at $12 \mathrm{hpi}$. (B) Lane 1, HSV-1 (F) BAC; lane 2, $\Delta \mathrm{UL} 43$. (C) Lane 1, gB $\Delta 28$; lane 2, gB $\Delta 28 / \Delta U L 43$. (D) Lane 1, gKsyn20; lane 2, gKsyn20/AUL43

the presence of the engineered mutations (Figure 3C, D).

\section{HSV-1 glycoproteins in infected cells}

A lack of UL43 expression was confirmed via the inability of anti-UL43 antibody to detect the presence of UL43 protein in the mutant viruses (Figure 4A). Overall, the expression levels of glycoproteins $\mathrm{gB}, \mathrm{gD}, \mathrm{gH}$ and $\mathrm{gL}$ were highly similar between the recombinant and HSV-1(F) BAC viruses, indicating that lack of UL43 did not affect the synthesis of viral glycoproteins.

\section{Cell surface expression of viral glycoproteins}

Deletion of UL43 did not affect overall expression levels of the viral glycoproteins $\mathrm{gB}, \mathrm{gD}, \mathrm{gH}$ and $\mathrm{gL}$ on HSV-1(F) BAC infected cell surfaces. Similar results were observed in the case of $\mathrm{gB} \Delta 28$ virus (Figure 4B-D).

\section{HSV-1 UL43 is required for efficient virus entry}

A significant delay in the rate of $\Delta U L 43$ virus entry was observed compared with the HSV-1(F) BAC (Figure 5). These observations suggested that UL43 protein is required for wild-type like virion entry, and the reduced entry rate of virions lacking UL43 could at least partly account for the defects in cell-to-cell spread of this virus.

\section{DISCUSSION}

HSV-1 enters cells predominantly via fusion of its viral envelope with cellular plasma membranes and can spread from infected to uninfected cells via virus-induced cell-to-cell fusion. Both membrane fusion phenomena are thought to be mediated by similar mechanisms involving glycoproteins gB, gD, gH, and $\mathrm{gL}$, which form a functional complex that is required for both virus entry and virus-induced cell fusion. The purpose 
of this study was to investigate whether the UL43 protein is involved in these membrane fusion phenomena. In this report, these data demonstrate a functional role of UL43 protein in regulating the HSV-1 membrane fusion during virus-induced cell fusion and virion entry.

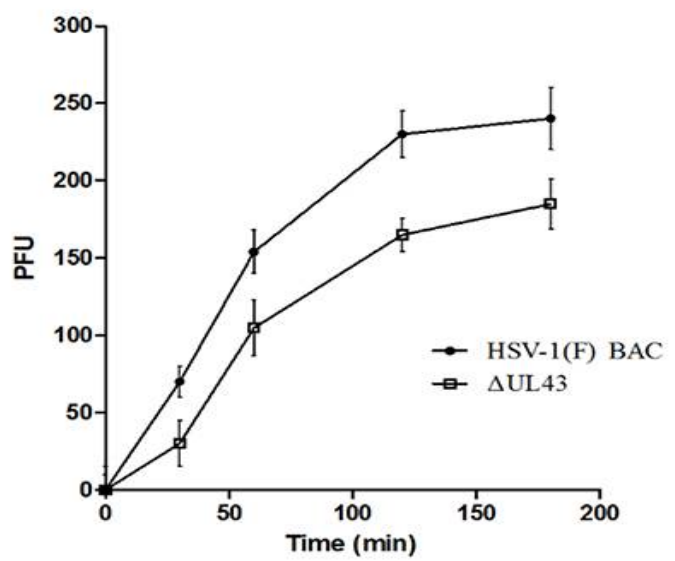

Figure 5: Entry kinetics of HSV-1(F) BAC and $\Delta U L 43$ viruses into Vero cells. And the linear regression slope were $2.10 \pm 0.09$ and $1.65 \pm 0.08$, respectively

The UL43 protein is highly hydrophobic and has been predicted to span membranes at least eight times (Figure 1A). Our detailed analysis of HSV1 UL43 deletion mutants confirmed previous findings that the UL43 protein is not required for virus growth in cell culture [15]. The BMRF2 protein, which is the UL43 homologous protein in Epstein-Barr-Virus (EBV), is involved in attachment and entry into polarized epithelial tongue and nasopharyngeal cells by binding to cellular integrin [16]. By comparison, a lack of HSV-1 UL43 was reported to have little effect in a mouse infection model [15], arguing against an essential role of UL43 in virus infection in vivo. Thus, studies in a natural virus-host system using appropriate mutant viruses are needed to be performed to solve this problem.

Although deletion of UL43 did not abrogate HSV1 replication, it significantly affected cell-to-cell spread and inhibited virus-induced cell fusion caused by a syncytial mutation in either $\mathrm{gB}$ or $\mathrm{gK}$. The $\mathrm{gB} \Delta 28$ mutation is the strongest $\mathrm{gB}$ syncytial mutation causing extensive cell fusion in tissue culture $[17,18]$. Unlike gB syncytial mutations, gKsyn20 mutations are known to cause extensive virus-induced cell fusion of all cell types, and gB syncytial mutations cause weaker fusion in a more limited repertoire of cells [19].

The experiments described herein have revealed that HSV-1 UL43 protein could be expressed on infected Vero cell surfaces and is required for virus-induced cell fusion. However, the PrV UL43 protein was suggested to inhibit membrane fusion in the transient co-expression system involving PrV envelope glycoproteins [10]. These findings seem to contradict results obtained herein with the recombinant viruses lacking UL43 gene. Interestingly, a similar result was observed for glycoprotein $\mathrm{gM}$ and $\mathrm{gK}$, which inhibited cell fusion, caused by transient co-expression of $\mathrm{gB}$, $\mathrm{gD}, \mathrm{gH}$, and $\mathrm{gL}$ [20-22] but were required for virus-induced cell fusion caused by syncytial mutations in $\mathrm{gB}$ [23]. These results indicate that UL43, as well as $\mathrm{gM}$ and $\mathrm{gK}$, may regulate virusinduced cell fusion both positively and negatively depending on the presence of different sets of viral proteins.

Transient coexpression of $\mathrm{gB}, \mathrm{gD}, \mathrm{gH}$, and $\mathrm{gL}$ is necessary and sufficient for the induction of cellto-cell fusion [24]. In spite of these Davis-Poynter et al concluded that UL43 are dispensable for syncytium formation [23], the recombinant viruses lacking the UL43 gene were constructed in the presence of strong syncytial mutations in glycoprotein B (gB) or gK. Lack of UL43 did not appear to appreciably impact the overall expression level of these viral glycoproteins or their relative levels expressed on infected cell surfaces, suggesting that UL43 may indirectly or physically interact with one or more of the viral glycoproteins to regulate cell fusion. Furthermore, it is possible that the roles of UL43 protein, $\mathrm{gM}$ and $\mathrm{gK}$ are redundant and related since all of these proteins represent multiple membrane spanning proteins.

The effect of UL43 protein on the highly complicated virus-induced cell fusion machinery remains largely unexplored and will be the subject of future experiments. In this regard, these results support the hypothesis that fusion of the viral envelope with cell membranes is similar to virus-induced cell fusion. A number of viral proteins are involved in virus-induced cell fusion machinery.

Thus, it is conceivable that deletion of viral membrane proteins other than $\mathrm{gB}, \mathrm{gD}, \mathrm{gH}$, and $\mathrm{gL}$ may affect viral entry, as has been shown here for UL43. Other membrane-associated proteins embedded in the viral envelope may exert site-specific regulation of membrane fusion phenomena mediated by fusogenic glycoproteins such as $\mathrm{gB}$, one of which may be the optimal recognition of various receptors and entry into different types of cells. 


\section{CONCLUSION}

Collectively, these results demonstrate an important role for HSV-1 UL43 protein in virusinduced cell fusion and efficient virus entry, although deletion of UL43 did not substantially affect virus replication in the cell culture. It is apparent that multiple functional interactions occur among viral glycoproteins and other envelope proteins that modulate membrane fusion phenomena in HSV-1 infections.

\section{ACKNOWLEDGEMENT}

This work was supported by the National Natural Science Foundation of China (NSFC no. 3087221), Guangdong Province Major Projects of Key Areas \{YSNo (2005) 162\}. Furthermore, the authors are grateful to $Y$ Mettenleiter for the gift of HSV-1 strain F.

\section{DECLARATIONS}

\section{Conflict of Interest}

No conflict of interest associated with this work.

\section{Contribution of Authors}

The authors declare that this work was done by the authors named in this article and all liabilities pertaining to claims relating to the content of this article will be borne by them.

\section{REFERENCES}

1. Cai WH, Gu B, Person S. Role of glycoprotein B of herpes simplex virus type 1 in viral entry and cell fusion. J Virol 1988; 62: 2596-2604.

2. Roop C, Hutchinson L, Johnson DC. A mutant herpes simplex virus type 1 unable to express glycoprotein $L$ cannot enter cells, and its particles lack glycoprotein $H$. J Virol 1993; 67: 2285-2297.

3. Spear PG, Eisenberg RJ, Cohen GH. Three classes of cell surface receptors for alphaherpesvirus entry. Virology 2000; 275: 1-8.

4. Baines JD, Ward PL, Campadelli-Fiume G, et al. The UL20 gene of herpes simplex virus 1 encodes a function necessary for viral egress. J Virol 1991; 65: 6414-6424.

5. Jacobson JG, Chen SH, Cook WJ, et al. Importance of the herpes simplex virus UL24 gene for productive ganglionic infection in mice. Virology 1998; 242: 161169.

6. Bzik DJ, Fox BA, DeLuca NA, et al. Nucleotide sequence of a region of the herpes simplex virus type $1 \mathrm{gB}$ glycoprotein gene: mutations affecting rate of virus entry and cell fusion. Virology 1984; 137: 185-190.
7. Bond VC, Person S. Fine structure physical map locations of alterations that affect cell fusion in herpes simplex virus type 1. Virology 1984; 132: 368-376.

8. Powers L, Wilkinson KS, Ryan P. Characterization of the PrV UL43 gene of pseudorabies virus and demonstration that it is not required for virus growth in cell culture. Virology 1994; 199: 81-88.

9. Carter KL, Ward PL, Roizman B. Characterization of the products of the UL43 gene of herpes simplex virus 1: potential implications for regulation of gene expression by antisense transcription. J Virol 1996; 70: 7663-7668.

10. Klupp BG, Altenschmidt J, Granzow H, et al. Identification and characterization of the pseudorabies virus UL43 protein. Virology 2005; 334: 224-233.

11. Tischer BK, von Einem J, Kaufer B, et al. Two-step redmediated recombination for versatile high-efficiency markerless DNA manipulation in Escherichia coli. Biotechniques 2006; 40: 191-197.

12. Lee HC, Chouljenko VN, Chouljenko DV, et al. The herpes simplex virus type 1 glycoprotein $D(g D)$ cytoplasmic terminus and full-length $g E$ are not essential and do not function in a redundant manner for cytoplasmic virion envelopment and egress. J Virol 2009; 83: 6115-6124.

13. Haugo AC, Szpara ML, Parsons L, et al. Herpes simplex virus 1 pUL34 plays a critical role in cell-to-cell spread of virus in addition to its role in virus replication. $J$ Virol 2011; 85: 7203-7215.

14. Klupp BG, Granzow H, Mundt E, et al. Pseudorabies virus UL37 gene product is involved in secondary envelopment. J Virol 2001; 75: 8927-8936.

15. MacLean CA, Efstathiou S, Elliott ML, et al. Investigation of herpes simplex virus type 1 genes encoding multiply inserted membrane proteins. J Gen Virol 1991; 72: 897906.

16. Tugizov SM, Berline JW, Palefsky JM. Epstein-Barr virus infection of polarized tongue and nasopharyngeal epithelial cells. Nat Med 2003; 9: 307-314.

17. Foster TP, Melancon JM, Kousoulas KG. An alpha-helical domain within the carboxyl terminus of herpes simplex virus type 1 (HSV-1) glycoprotein $B(g B)$ is associated with cell fusion and resistance to heparin inhibition of cell fusion. Virology 2001; 287: 18-29.

18. Silverman JL, Greene NG, King DS, et al. Membrane requirement for folding of the herpes simplex virus $1 \mathrm{gB}$ cytodomain suggests a unique mechanism of fusion regulation. J Virol 2012; 86: 8171-8184.

19. Manservigi R, Spear PG, Buchan A. Cell fusion induced by herpes simplex virus is promoted and suppressed by different viral glycoproteins. Proc Natl Acad Sci U S A 1977; 74: 3913-3917.

20. Avitabile E, Lombardi G, Campadelli-Fiume G. Herpes simplex virus glycoprotein $K$, but not its syncytial allele, inhibits cell-cell fusion mediated by the four fusogenic glycoproteins, $g D, g B, g H$, and $g L$. J Virol 2003; 77: 6836-6844. 
21. Koyano S, Mar EC, Stamey FR, et al. Glycoproteins $M$ and $N$ of human herpesvirus 8 form a complex and inhibit cell fusion. J Gen Virol 2003; 84: 1485-1491.

22. Klupp BG, Nixdorf R, Mettenleiter TC. Pseudorabies virus glycoprotein M inhibits membrane fusion. J Virol 2000; 74: 6760-6768.

23. Davis-Poynter N, Bell S, Minson T, et al. Analysis of the contributions of herpes simplex virus type 1 membrane proteins to the induction of cell-cell fusion. J Virol 1994; 68: 7586-7590.

24. Turner A, Bruun B, Minson T, et al. Glycoproteins $g B, g D$, and $\mathrm{gHgL}$ of herpes simplex virus type 1 are necessary and sufficient to mediate membrane fusion in a Cos cell transfection system. J Virol 1998; 72: 873-875. 